\title{
Training Requirements for Theranostics: A Unique Opportunity for Collaboration
}

\author{
Michael M. Graham ${ }^{1}$ and John M. Buatti ${ }^{2}$ \\ ${ }^{1}$ Department of Radiology, University of Iowa, Iowa City, Iowa; and ${ }^{2}$ Department of Radiation Oncology, University of Iowa, Iowa \\ City, Iowa
}

$\mathbf{T}$

he current training programs relevant for theranostic therapy include nuclear medicine and radiation oncology. Each has independent residencies of several years duration with accredited board certifications. Nuclear medicine is focused on the use of radioisotopes for both diagnostic and therapeutic applications, with prominent emphasis on radiochemistry, radiopharmacy management, human radiopharmaceutical metabolism, and pharmacokinetics. Radiation oncology is focused on oncologic care and decision making, with details of treatment simulation for individualized cancer patient dosimetry, radiation toxicity, and tumoricidal effects on tissues (cells to organs) with attention to volume, dose fractionation, and outcomes in follow-up cancer care. Both training programs have requirements addressing radiation safety and tailored radiation biology knowledge. Both specialties routinely deliver a range of radioisotope therapies.

Within this context, a rapidly evolving and indeed disruptive advance of therapeutically relevant theranostic agents is on us. For decades, radioiodine therapy for thyroid cancer and hyperthyroid thyrotoxicosis has been the only form of radiopharmaceutical theranostic. ${ }^{123}$ I and ${ }^{131}$ I have been used for identification of metastatic disease and for assisting in the calculation of the administered therapeutic dose of ${ }^{131} \mathrm{I}$. More recently, the theranostic pair of ${ }^{68} \mathrm{Ga}$-DOTATATE and ${ }^{177} \mathrm{Lu}$-DOTATATE has become commercially available, and now patients with neuroendocrine tumors are being evaluated with the former and treated with the latter (1). ${ }^{68} \mathrm{Ga}$-PSMA and ${ }^{177} \mathrm{Lu}$-PSMA will soon become available for the evaluation and treatment of prostate cancer (2). Multiple other promising theranostic pairs are under development and in the next few years will become available for administration to patients. Advances in biologically targeted chemistry appear to be translating positively into a new era for theranostics $(3,4)$.

Our training and paradigms for care delivery have not really been optimized for the best theranostic oncology patient care. Currently, physicians who are authorized to administer these therapeutic agents are "authorized users," a designation from the $\mathrm{Nu}$ clear Regulatory Commission, which requires specified training and experience, including experience in the administration of parenteral therapeutic radiopharmaceuticals in at least 5 patients. These physicians have been trained in the specialties of nuclear

Received Mar. 21, 2019; revision accepted Mar. 26, 2019.

For correspondence or reprints contact: Michael M. Graham, Department of Radiology, University of lowa, 200 Hawkins Dr., 3863 JPP, lowa City, IA 52242.

E-mail: michael-graham@uiowa.edu

Published online Apr. 6, 2019.

COPYRIGHT (C) 2019 by the Society of Nuclear Medicine and Molecular Imaging.

DOI: 10.2967/jnumed.119.228940 medicine or radiation oncology. The training programs are not identical, but both include radiation safety, radiobiology, and significant experience in treating thyroid cancer with ${ }^{131} \mathrm{I}$. Because ${ }^{68} \mathrm{Ga} /{ }^{177} \mathrm{Lu}$-DOTATATE has become available only recently, there are no specifically applicable training requirements. Furthermore, ${ }^{177} \mathrm{Lu}$-DOTATATE treatment of neuroendocrine tumors is significantly more complex than the treatment of thyroid cancer, and it remains unclear whether this therapy has been optimized in terms of individualized dosimetry and dosing for best patient care and outcome $(5,6)$. Additional training, as well as enhanced collaboration between our specialties, will be required to conduct these new theranostic therapies most effectively for the optimal care of patients.

Nuclear medicine physicians, who have been primarily diagnosticians, will have to assume the role of clinical oncologist in the management of cancers and also, of particular importance, learn to manage treatment responses and adverse reactions. They will need to learn more about the pathophysiology of the relevant malignancies, the effects of alternative or concurrent treatments, and the enhanced dosimetry required to select the most effective administered dose and to avoid normal-organ injury. Radiation oncologists will need to learn more about radiopharmacy practices, radiopharmaceutical metabolism, and pharmacokinetics, as well as new radiation safety requirements for radioactive patients treated with a range of new agents. The methods for individualized, accurate, internal dosimetry are important, are rapidly evolving, and will require a concerted effort from our physics colleagues in both nuclear medicine and radiation oncology.

Listed below are the most important areas of knowledge and experience that theranostic physician teams (including residents and practicing physicians) will need to master to provide the best theranostic oncologic care. Physicians need to thoroughly evaluate the need for therapy with knowledge of alternative oncologic therapies. They will need to determine the optimal theranostic agent and pharmacokinetic parameters to calculate an individual patient's optimized administered dose. They will need to supervise the process for theranostic agent preparation, quality assurance, and administration. Finally, the number and timing of treatments will need to be prescribed, and the plan for follow-up assessment and care of patients receiving this therapy will need to occur.

- Pathobiology of relevant oncologic diseases

- Broad understanding of the alternative therapy options

- Indications for theranostic treatment

- Initial workup requirements and potential contraindications and therapy considerations, including previous treatments with radiation or chemotherapy affecting organ function (marrow reserve, kidney function)

- Pretherapy imaging 
- Pretherapy medication

- Basics of the infusion procedure

- Dosimetry calculation and uncertainty considering both target doses and organs at risk

- Posttherapy imaging

- Possible adverse events and their management, both acutely and considering late effects

- Appropriate blood chemistry and its interpretation both before and after therapy

- Appropriate follow-up visits, examinations, and imaging for response and toxicity outcomes

This list is not intended to be comprehensive but should provide a starting point for determining how to modify current existing residency training programs, as well as training for practicing physicians, in light of the exciting expansion in theranostic oncology therapy. How to change the formal requirements as stated by the Residency Review Committees and the boards, and determination of what type of continuing education needs to be provided to practicing physicians, should be guided by establishing a model that prioritizes the best care for cancer patients and leverages the unique and complementary knowledge base within nuclear medicine and radiation oncology.

\section{DISCLOSURE}

No potential conflict of interest relevant to this article was reported.

\section{REFERENCES}

1. Fani M, Nicolas GP, Wild D. Somatostatin receptor antagonists for imaging and therapy. J Nucl Med. 2017;58(suppl 2):61S-66S.

2. Rahbar K, Afshar-Oromieh A, Jadvar H, Ahmadzadehfar H. PSMA theranostics: current status and future directions. Mol Imaging. 2018;17:1536012118776068.

3. Ballinger JR. Theranostic radiopharmaceuticals: established agents in current use. Br J Radiol. 2018;91:20170969.

4. Jadvar H. Targeted radionuclide therapy: an evolution toward precision cancer treatment. AJR. 2017;209:277-288.

5. Besemer AE, Yang YM, Grudzinski JJ, Hall LT, Bednarz BP. Development and validation of RAPID: a patient-specific Monte Carlo three-dimensional internal dosimetry platform. Cancer Biother Radiopharm. 2018;33:155-165.

6. Santoro L, Mora-Ramirez E, Trauchessec D, et al. Implementation of patient dosimetry in the clinical practice after targeted radiotherapy using $\left[{ }^{177} \mathrm{Lu}\right.$-[DOTA0, Tyr3]-octreotate. EJNMMI Res. 2018;8:103. 